\title{
Bi-directional impulse chaos control in crystal growth
}

\author{
Zi-Xuan Zhou, ${ }^{1,2}$ Hai-Peng Ren, ${ }^{1,}$ a) and Celso Grebogi ${ }^{1,3}$ \\ ${ }^{1)}$ Shaanxi Key Laboratory of Complex System Control and Intelligent Information Processing, Xi'an University of Technology, \\ Xi'an, Shaanxi 710048, China; \\ ${ }^{2)}$ School of Computer Science and Engineering, North Minzu University, Yinchuan, Ningxia 750021, \\ China \\ ${ }^{3)}$ Institute for Complex System and Mathematical Biology, King's College, University of Aberdeen, Aberdeen AB24 $3 U E$, \\ United Kingdom
}

(Dated: 9 April 2021)

\begin{abstract}
Mono-silicon crystals, free of defects, are essential for the Integrated Circuit industry. Chaotic swing in the flexible shaft rotating-lifting (FSRL) system of the mono-silicon crystal puller causes harm to the quality of the crystal and must be suppressed in the crystal growth procedure. From the control system viewpoint, the constraints of the FSRL system can be summarized as not having measurable state variables for state feedback control, and only one parameter is available to be manipulated, namely, the rotation speed. From the application side, an additional constraint is that the control should affect the crystallization physical growth process as little as possible. These constraints make the chaos suppression in the FSRL system to be a challenging task. In this work, the analytical periodic solution of the swing in the FSRL system is derived using perturbation analysis. A bi-directional impulse control method is then proposed for suppressing chaos. This control method does not alter the average rotation speed. It is thus optimum regarding the crystallization process as compared with the single direction impulse control. The effectiveness and robustness of the proposed chaos control method to parameters uncertainties are validated by the simulations.
\end{abstract}

Mono-silicon crystal is the basic material of Integrated Circuits and solar panels, which is grown by using a mono-silicon crystal puller in a 1420 Celsius degree airtight vacuum environment. During the crystal growth procedure, the flexible shaft rotating-lifting (FSRL) system keeps both the crystal rotating smoothly and the gradual crystal pulling, according to the crystallization physical requirements. In such a way, the atoms in the silicon melt within the crucible are able to crystalize in a predefined array that ensures crystal quality. However, an irregular and chaotic swing of the FSRL system, caused by external perturbation, is present. That must be suppressed since it does harm the crystal quality. The dynamics and the presence of chaos in the FSRL system was first investigated in [1]. The irregular swing has been identified for some time, traditionally considered as being part of an imperfect manufacturing. However, improvements in manufacturing could not solve the problem. Furthermore, with the increase demand in the diameter of the crystal, the height of the puller and the length of the shaft have become larger, making the situation even worse. To solve the problem, engineers replaced the soft shaft with a hard shaft, so that the swing could be eliminated. Although this solution is somewhat effective, it comes at high cost, making it often a too expensive solution for such competitive industry. For this reason and after the dynamics being understood as a chaotic vibration problem [1], there is the possibility to solve the problem by chaos (vibration) control methods. There exist much theoretical research about chaos control. However, when facing with a practical plant, most of them are excluded due to operation constraints. The constraints in the FSRL system can be summarized as: (i)

\footnotetext{
a)Electronic mail: renhaipeng@xaut.edu.cn
}

no state feedback is available; (ii) only the rotation speed parameter is accessible for manipulation; (iii) the control method must have no or little effect on the crystallization process. Facing with these constraints, the state feedback based methods are outrightly excluded. Therefore, for a functioning plant, the control of chaos is not a trivial task under such restrictive constraints. The periodic single impulse control is a candidate for the FRSL system [2]. However, there exists a significant weak point in that control method. It leads the average rotation speed, which is the controlled parameter, to deviate from the physical requirements for the crystallization. To address this shortcoming, a bi-directional impulse control is being proposed in this work. The proposed method keeps the merits of impulse control, i.e., the application of small perturbations to the manipulating parameter, while keeping the average rotation speed constant. The controlling parameter perturbations are determined according to a bifurcation analysis. The select range is verified by numerical simulations. The vibration response is analyzed using a singular perturbation method. The robustness of the FSRL system due to parameters uncertainties is also validated by simulations.

\section{INTRODUCTION}

Mono-silicon crystal puller is a manufacturing large apparatus in which polycrystalline silicon blocks are melted in a crucible using graphite heater. From a mono-silicon seed, a mono-silicon crystal is grown in an inert gas vacuum environment. The large mono-silicon crystals play an important role in semiconductor integrated circuits and solar panels industry. An undesirable irregular swing has been an impediment during the seeding stage in the FSRL system [1]. It is found that the irregular swing, associated with chaos, disrupts the atoms 


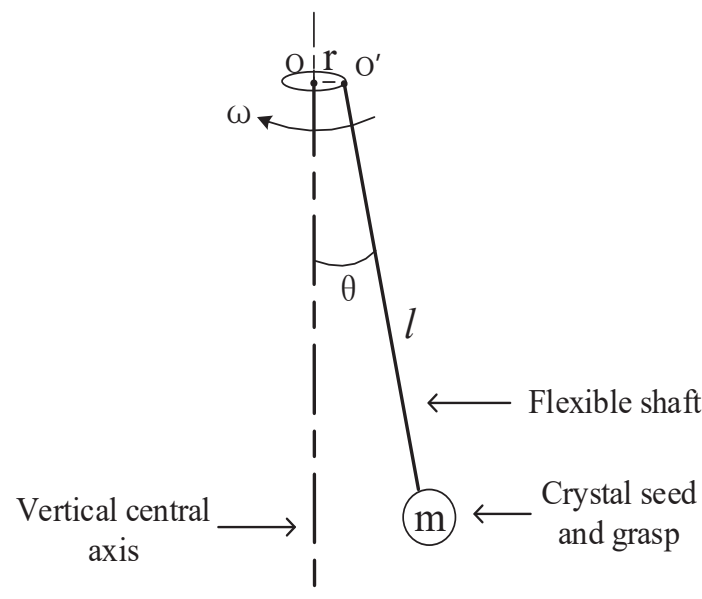

FIG. 1. The simplified structure diagram of the FSRL system.

to crystalize in a predefined and perfect array, causing defective mono-silicon crystal production. Therefore, the chaotic swing must be suppressed. Due to the lack of understanding about the intrinsic chaotic dynamics of the FSRL system in the past, the field engineers attributed the irregular swing phenomenon to the imperfections in manufacturing, trying to suppress it by fine mechanical adjustments. However, these methods could not eliminate the swing phenomenon, intrinsic to the nonlinear dynamics of the FSRL system as systematically analyzed in [1]. Thus, chaos control methods have been recognized as a solution to suppress the irregular swing.

The crystal seed having $10 \mathrm{~mm}$ in diameter is grasped and fixed at the end of a flexible shaft, as shown in Fig. 1. The other end of the flexible shaft is fixed in a rotating disk. During the process of the mono-silicon crystal production, the rotating disk, driven by a motor, rotates at a speed determined by the crystallization physical requirements. In ideal conditions, the crystal seed rotates in the melted silicon in a period one mode and is lifted slowly, so that the crystal is grown to a larger diameter with a predefined atom array. However, the small apparatus eccentricity brings about a small external perturbation to the FSRL system, leading to the observed chaotic phenomenon. In order to suppress chaos in the manufacturing plant, the following practical constraints have to be considered: (i) no state variable can be measured for controller design; (ii) the only accessible parameter is the rotation speed; (iii) the controlling perturbation must be as small as possible as required by the physics of the crystal growth. Bearing all the above constraints in mind, we consider the chaos control methods reported since the seminal work by Ott, Grebogi and Yorke [3]. Because there is no measurable system state of the FSRL system, all state feedback based methods are excluded, such as linear feedback [4-5], delay feedback [6], adaptive control [7-8], active control [9], robust control [10], and fuzzy control [11]. Anishchenko's work on chaos synchronization, as a kind of chaos control method, yielded fruitful research results [12-14], but it cannot be used to suppress chaos in the FSRL system. The random phase perturbation method has been proposed to control chaos in Duffing oscillator [15], however, for FSRL system with the similar dynamic equation as that of the oscillator in [15], there is no way to perturb the eccentric phase of the FSRL system, because of the physical constraints given before. Fortunately, periodic impulses can stabilize chaos by perturbing a system parameter at every small interval, having less influence on the steady state of the controlled system.

In general, the impulse control method is based on the theory of impulsive differential equations. Researchers studied the asymptotic stability of the impulse control system by using the Lyapunov method [16-18] and the comparison theorem [19]. Among the published impulse control methods, some control strategies require to adjust all state variables [20-22], others achieve the goal by manipulating only one state variable [2324], but only [25] dealt with the perturbation of a parameter, as required in our work. In addition, depending on the different requirements for the impulse action of the control system, the impulse could be applied at a fixed interval [26-27], or at a state-dependent one [28-29]. An impulse control method was proposed for suppressing the chaos in Duffing oscillator in [30], however, the method in [30] put the impulse on the oscillation force, which is impossible for a physical system like FSRL, where the physical constraints have to be obeyed. For a practice system in which the state variables cannot be measured and the manipulated variable is firmly restricted, such as the FSRL system, the parameter impulse control is the a appropriate control method. Hence, a single directional parameter impulse control was proposed to perturb the rotation speed in [2]. However, there is a drawback in that method. The single direction impulse alters the average rotation speed (although the change is small). In practice, this rotation speed is determined by the delicate crystal growth physical requirements, which cannot be altered. To overcome this drawback, a bi-directional impulse control method is being proposed in this work. The key characteristic of this method is that the average rotation speed is kept the same as the unperturbed one, representing an essential feature for the manufacturing industry.

The organization of this paper is as follows. In Section 2, the dynamics of the FSRL system is revisited, and the vibration response of the system is obtained by using a singular perturbation method. In Section 3, a bi-directional impulse control method is proposed to suppress chaotic motion in the FSRL system and the control parameters selection rule is given. In Section 4, the simulation results are given to show the effectiveness of the proposed method. The robustness of the proposed method to parameter uncertainties are also analyzed. In Section 5, conclusions are given.

\section{NONLINEAR DYNAMICS AND VIBRATION RESPONSE ANALYSIS OF THE FSRL SYSTEM}

A simplified schematic diagram of the FSRL system is given in Fig. 1. The system is governed by the following equation:

$$
\ddot{\theta}=\frac{r}{l} \omega^{2} \cos (\omega t)+\omega^{2} \sin \theta \cos \theta-\frac{g}{l} \sin \theta-\frac{\xi}{m} \dot{\theta},
$$




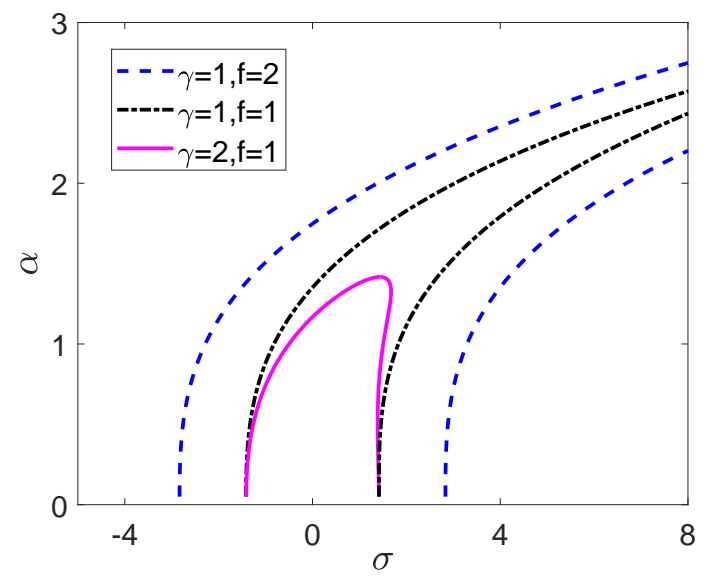

FIG. 2. The amplitude-frequency response of the FSRL system for different parameter sets. $\alpha$ represents the amplitude response of the system and $\sigma$ represents the frequency near the main parametric resonance. The blue dashed line corresponds to the parameters $\gamma=1, f=2$; the black dash dotted line corresponds to the parameters $\gamma=1, f=1$; the magenta solid line corresponds to the parameters $\gamma=2, f=1$.

where $\theta$ is the angle between the rotational axis and the shaft, $\omega$ is the rotation speed, $l$ is the length of flexible shaft from the fixed point $O^{\prime}$ to the crystal seed, $m$ is the mass of the crystal seed and the grasp, $\xi$ is the damping coefficient, and $g$ is the gravitational acceleration. A motor drives the rotating disk with a belt pulley. The eccentricity effect causes the periodic perturbation of the fixed point $O, r$ being the distance from the eccentric fixed point $O^{\prime}$ to the shaft central point $O$. The working principle and modeling of the FSRL system have been investigated in [1].

The perturbation analysis of the system is conducted using multiple scales method. Because the coefficients $\frac{r}{l}$ and $\frac{\xi}{m}$ in the dynamical equation are very small, they can be represented in terms of a small parameter $\varepsilon$. Expanding $\sin \theta$ in a Taylor series and retaining the first two terms, Eq. (1) can be rewritten as follows,

$$
\ddot{\vartheta}+\omega_{n}^{2} \vartheta+\left(\frac{1}{2} \omega^{2}-\frac{1}{6} \omega_{n}^{2}\right) \varepsilon \vartheta^{3}+\varepsilon \gamma \dot{\vartheta}=\varepsilon f \omega^{2} \cos (\omega t),
$$

where $\omega_{0}^{2}=\frac{g}{l}, \sqrt{\varepsilon^{3}} f=\frac{r}{l}, \varepsilon \gamma=\frac{\xi}{m}, \omega_{n}^{2}=\omega_{0}^{2}-\omega^{2}$, and $\theta=$ $\sqrt{\varepsilon} \vartheta$.

The general solution of Eq. (2) is assumed to be of the form, $\vartheta=\vartheta_{0}+\varepsilon \vartheta_{1}+\cdots$. A series of independent variables are introduced: $T_{0}=\tau, T_{1}=\varepsilon \tau, \cdots, T_{n}=\varepsilon^{n} \tau$, which are the time scales, so that $\vartheta$ is the function $\vartheta\left(T_{0}, T_{1}, \cdots\right)$. The time derivatives are given by the chain rule,

$$
\frac{d}{d \tau}=D_{0}+\varepsilon D_{1}+\varepsilon^{2} D_{2}+\cdots
$$

and

$$
\frac{d^{2}}{d \tau^{2}}=D_{0}^{2}+2 \varepsilon D_{0} D_{1}+\varepsilon^{2}\left(2 D_{0} D_{2}+D_{1}^{2}\right)+\cdots
$$

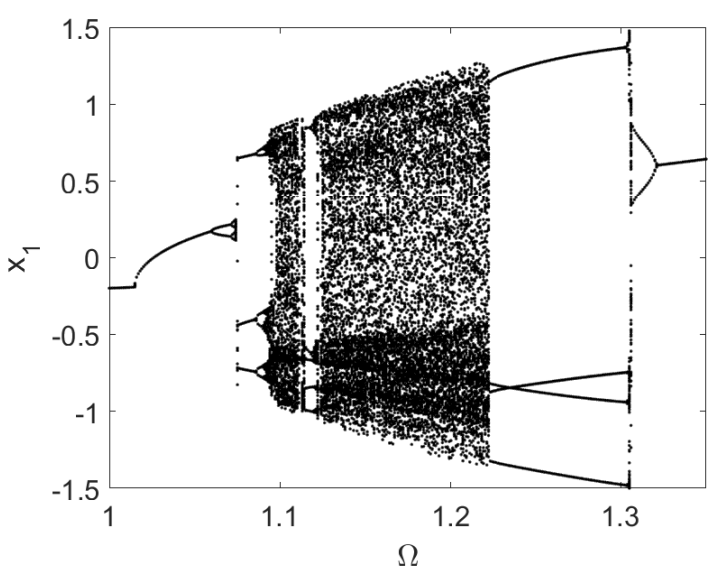

FIG. 3. The bifurcation diagrams of the system (16) for $A=0.2, c=$ 0.1 .

where $D_{n}^{m}=\partial^{m} / \partial T_{n}^{m}$. In the following, the general solution and its time derivatives are substituted into Eq. (2). By grouping together the terms with the same powers of $\varepsilon$, the set of equations up to order $\varepsilon$ are as follows,

$$
\varepsilon^{0}: D_{0}^{2} \vartheta_{0}+\omega_{n}^{2} \vartheta_{0}=0
$$

and

$$
\begin{aligned}
\varepsilon^{1}: D_{0}^{2} \vartheta_{1}+\omega_{n}^{2} \vartheta_{1} & =-2 D_{0} D_{1} \vartheta_{0}-\gamma D_{0} \vartheta_{0} \\
& -\left(\frac{1}{2} \omega^{2}-\frac{1}{6} \omega_{n}^{2}\right) \vartheta_{0}^{3}+f \omega^{2} \cos (\omega t)
\end{aligned}
$$

The solution of Eq. (5) is the following,

$$
\begin{aligned}
\vartheta_{0} & =\alpha \cos \left(\omega_{n} T_{0}+\beta\right) . \\
& =B e^{i \omega_{n} T_{0}}+\bar{B} e^{-i \omega_{n} T_{0}},
\end{aligned}
$$

where $B=\frac{1}{2} \alpha e^{i \beta}, \bar{B}=\frac{1}{2} \alpha e^{-i \beta}, \bar{B}$ is the complex conjugate of $B$. For the first approximation solution, it can be assumed that $\alpha=\alpha\left(T_{1}\right), \beta=\beta\left(T_{1}\right), B=B\left(T_{1}\right), \bar{B}=\bar{B}\left(T_{1}\right)$.

Substituting Eq. (7) into Eq. (6), we have

$$
\begin{aligned}
D_{0}^{2} \vartheta_{1}+\omega^{2} \vartheta_{1} & =\left(\frac{1}{6} \omega_{n}^{2}-\frac{1}{2} \omega^{2}\right)\left(B e^{i \omega_{n} T_{0}}+\bar{B} e^{-i \omega_{n} T_{0}}\right)^{3} \\
& -2 D_{1}\left(i \omega_{n} B e^{i \omega_{n} T_{0}}-i \omega_{n} \bar{B} e^{-i \omega_{n} T_{0}}\right) \\
& -\gamma\left(i \omega_{n} B e^{i \omega_{n} T_{0}}-i \omega_{n} \bar{B} e^{-i \omega_{n} T_{0}}\right) \\
& +f \omega^{2} \cos (\omega t) .
\end{aligned}
$$

By introducing a detuning parameter $\sigma$, which is expressed as $\omega=\omega_{n}+\varepsilon \sigma$, the following equation is obtained for the time scale $T_{1}$,

$$
\cos (\omega t)=\cos \left(\omega_{n} T_{0}+\sigma T_{1}\right) .
$$

Using Euler's formula, Eq. (9) can be written in complex 
conjugate form, and Eq. (8) becomes,

$$
\begin{aligned}
D_{0}^{2} \vartheta_{1}+\omega^{2} \vartheta_{1} & =\left(\frac{1}{6} \omega_{n}^{2}-\frac{1}{2} \omega^{2}\right) B^{3} e^{3 i \omega_{n} T_{0}} \\
& +\left(\frac{1}{6} \omega_{n}^{2}-\frac{1}{2} \omega^{2}\right) \bar{B}^{3} e^{-3 i \omega_{n} T_{0}} \\
& +\left(\frac{1}{2} \omega_{n}^{2}-2 \omega^{2}\right) B^{2} \bar{B} e^{i \omega_{n} T_{0}} \\
& +\left(\frac{1}{2} \omega_{n}^{2}-2 \omega^{2}\right) B \bar{B}^{2} e^{-i \omega_{n} T_{0}} \\
& -2 i \omega_{n} D_{1} B e^{i \omega_{n} T_{0}}+2 i \omega_{n} D_{1} \bar{B} e^{-i \omega_{n} T_{0}} \\
& -i \gamma \omega_{n} B e^{i \omega_{n} T_{0}}+i \gamma \omega_{n} \bar{B} e^{i \omega_{n} T_{0}} \\
& +\frac{f \omega^{2}}{2} e^{i \omega_{n} T_{0}+i \sigma T_{1}}+\frac{f \omega^{2}}{2} e^{-i \omega_{n} T_{0}-i \sigma T_{1}}
\end{aligned}
$$

Because the complex conjugate terms with $e^{i \omega_{n} T_{0}}$ and $e^{-i \omega_{n} T_{0}}$ are secular terms, they must be eliminated by setting the coefficients to zero. Thereby, the following condition is obtained,

$$
\left(\frac{1}{2} \omega_{n}^{2}-\frac{3}{2} \omega^{2}\right) B^{2} \bar{B}-i \gamma \omega_{n} B-2 i \omega_{n} D_{1} B+\frac{f \omega^{2}}{2} e^{i \sigma T_{1}}=0 .
$$

Substituting $B=\frac{1}{2} \alpha e^{i \beta}, \quad \bar{B}=\frac{1}{2} \alpha e^{-i \beta}, \quad$ and $e^{i \sigma T_{1}}=$ $\cos \left(\sigma T_{1}-\beta\right)+i \sin \left(\sigma T_{1}-\beta\right)$ into Eq. (11), we have

$$
\begin{aligned}
\left(\frac{1}{2} \omega_{n}^{2}\right. & \left.-\frac{3}{2} \omega^{2}\right) \times \frac{1}{8} \alpha^{3}-\frac{1}{2} i \omega_{n} \gamma \alpha-i \omega_{n} D_{1} \alpha+\omega_{n} D_{1} \beta \\
+ & \frac{f \omega^{2}}{2} \cos \left(\sigma T_{1}-\beta\right)+i \frac{f \omega^{2}}{2} \sin \left(\sigma T_{1}-\beta\right)=0
\end{aligned}
$$

By introducing an auxiliary variable $\varphi=\beta-\sigma T_{1}$, and splitting Eq. (12) into the real and imaginary parts, we obtain the expressions for $D_{1} \alpha$ and $D_{1} \varphi$ as given by

$$
D_{1} \alpha=-\frac{1}{2} \gamma \alpha-\frac{f \omega^{2}}{2 \omega_{n}} \sin \varphi
$$

and

$$
D_{1} \varphi=-\sigma-\left(\frac{1}{16} \omega_{n}^{2}-\frac{3}{16} \omega^{2}\right) \times \frac{\alpha^{3}}{\omega_{n}}-\frac{f \omega^{2}}{2 \omega_{n}} \cos \varphi .
$$

When $\alpha$ and $\varphi$ are in the steady state, $D_{1} \alpha=0$ and $D_{1} \varphi=0$. Since $\sin ^{2} \varphi+\cos ^{2} \varphi=1$, the amplitude-frequency response equation is obtained as,

$$
\left(\frac{1}{2} \gamma \alpha\right)^{2}+\left(\sigma+\left(\frac{1}{16} \omega_{n}^{2}-\frac{3}{16} \omega^{2}\right) \times \frac{\alpha^{3}}{\omega_{n}}\right)^{2}=\left(\frac{f \omega^{2}}{2 \omega_{n}}\right)^{2} .
$$

The amplitude-frequency response curves for different parameters are shown in Fig. 2. The system amplitude response increases with the increase of the excitation frequency $f$, while, with the increase of the damping coefficient, the system amplitude response decreases gradually.

Importantly, the curves of the amplitude frequency response reveal the nonlinear features of the FSRL system, such as the inclination and jump phenomena. The green and black curves bending over to the right indicate that the same frequency corresponds to two amplitude values, meaning system instability. The magenta curve represents the case where the system damping is sufficiently large. The amplitude response of the system is greatly reduced, and the inclination and jump phenomena disappear. However, in industrial plant, the system damping, though small, cannot be manipulated.

As studied in [1], the FSRL system exhibits various dynamical behaviors, including period doubling bifurcation, symmetry-breaking bifurcation, interior crisis, and chaotic motion.

To see that, we rewrite system (1) into a dimensionless for$\mathrm{m}$,

$$
\begin{aligned}
& \dot{x_{1}}=x_{2}, \\
& \dot{x_{2}}=A \Omega^{2} \cos (\Omega t)+\Omega^{2} \sin x_{1} \cos x_{1}-\sin x_{1}-c x_{2},
\end{aligned}
$$

where $x_{1}=\theta, x_{2}=\dot{\theta}, A=\frac{r}{l}, c=\frac{\xi}{m} . A$ and $\Omega$ are the amplitude and frequency of the excitation, respectively.

Figure 3 shows the bifurcation diagram of system (16) for $A=0.2, c=0.1$, chaos occurs when $\Omega \in(1.08,1.23)$. It demonstrates the nonlinear dynamics of the system. For additional dynamical analysis, we refer to [1].

\section{A BI-DIRECTIONAL IMPULSE CONTROL METHOD OF THE FSRL SYSTEM}

In this Section, a bi-directional impulse control method is proposed for suppressing chaos in the FSRL system. The main goal of the approach is to eliminate chaotic motion while maintaining the average rotation speed to be constant. Because the rotation speed is selected as the control input, we define positive impulse when the speed increases and negative impulse as it decreases. The dimensionless equation of the proposed bi-directional controlled system is as follows:

$$
\begin{aligned}
\dot{x_{1}} & =x_{2}, \\
\dot{x_{2}} & =A(P(t))^{2} \cos \left((P(t) t)+(P(t))^{2} \sin x_{1} \cos x_{1}\right. \\
& -\sin x_{1}-c x_{2},
\end{aligned}
$$

where $P(t)=\Omega+u(t)$ is determined by the rotation speed. $u(t)$ is defined as the impulse function with period $T=2 \pi / \Omega$, which is expressed as:

$$
u(t)=\sum_{n=0}^{\infty} h(t-n T)
$$

with

$$
h(t)= \begin{cases}-\kappa, & 0<t<\Delta \\ \kappa, & T / 2<t<T / 2+\Delta \\ 0, & \text { otherwise, }\end{cases}
$$

where $\Delta$ is the impulse duration and $\kappa$ is the impulse amplitude. From Eqs. (18) and (19), we require that both impulses are applied to the rotation speed sequentially. The positive $\kappa$ 


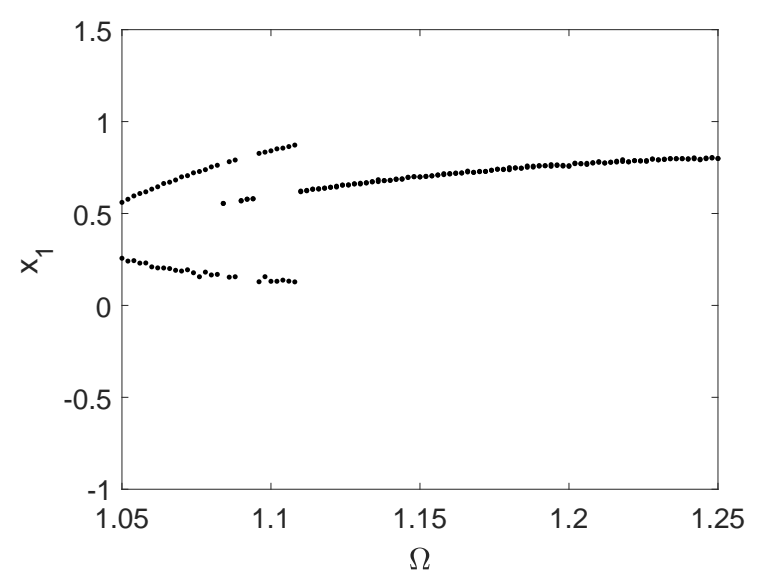

FIG. 4. The bifurcation diagrams of $x_{1}$ versus $\Omega$ for the controlled system (17) with parameter pair $(\kappa, \Delta)=(0.8,0.342)$.

means that the speed is increasing and the negative $\kappa$ means that the speed is decreasing. The bi-directional impulse control alternatively increases and decreases the speed within duration $\Delta$ at each half period of the rotation.

Figure 4 shows the bifurcation diagram of the controlled system (17) using bi-directional impulse control, in which the asymptotic state variable $x_{1}$ is plotted against the rotation speed $\Omega$. It represents the periodic motion after the bidirectional impulse control is applied, where the control parameters are $(\kappa, \Delta)=(0.8,0.342)$. The impulse duration $\Delta$ is 6 percent of the period, $2 \pi / \Omega$. Comparing with Fig. 3 , it can be seen from Fig. 4 that the chaotic motion is converted into a periodic motion by the proposed bi-directional impulse control.

The bifurcation diagrams of the controlling impulse parameters are shown in Figs. 5(a) and 5(b) for $\kappa$ and $\Delta$, respectively. Figure 5(a) shows that the state $x_{1}$ of the controlled system (17) changes from chaotic motion to period one motion for $\kappa \in(0.58,1)$ when $\Delta$ is fixed at 0.285 . In Fig. 5(b), $\kappa$ is fixed at 0.6 , the state $x_{1}$ is periodic for $\Delta \in(0.057,0.4)$. Therefore, the bi-directional impulse control with the appropriate parameter pair $(\kappa, \Delta)$ does eliminate the chaotic motion in the controlled system.

In the mono-silicon growth process, a stable rotation between the seed and the melted silicon is essential to achieve stable crystal/melt surface shape. Considering the special requirements for the crystal growth technology, we determine the parameter boundary for the controlled system to operate in a stable period one motion, as shown in Fig. 6. With our control strategy, the system continues to operate in the period one motion. In Fig. 6, chaos is suppressed by using the bidirectional impulse control with parameter pairs $(\kappa, \Delta)$ in the shade area, where the system parameters $\Omega=1.1, A=0.2$ and $c=0.1$. For other system parameters, the control parameter boundary can also be determined similarly.

\section{SIMULATION RESULTS}

\section{A. The effectiveness of the bi-directional impulse control method}

In the following, we verify the effectiveness of the proposed method by using simulations. The parameters selection, according to the rule given above, ensures that the chaotic behavior of the system is suppressed and the system is stabilized at period one.

The bi-directional impulse control results are shown in Fig. 7 (a) for the parameter pair $(\kappa, \Delta)=(0.8,0.257)$ and the system parameters $\Omega=1.1, A=0.2, c=0.1$. Figure 7(a) depicts the waveform of the state $x_{1}$ of the controlled system (17), where the control impulses are activated at $t=100$. It shows the state $x_{1}$ transition from chaotic motion to period one after $t=100$.

We compare the single direction impulse control with the bi-directional impulse control. The equation of the controlled system for the single impulse control method is defined in [2]. The method applies one negative impulse per period. Figure 7(b) shows the simulation result of single impulse control for choosing the control parameters pair $(\kappa, \Delta)=(-0.8,0.51)$ and the system parameters $\Omega=1.1, A=0.2, c=0.1$. The impulse amplitude of single impulse control is the same as that of bidirectional impulse control, while the impulse duration of these two control methods are also the same. Comparing with Fig. 7(a), it can be seen that the amplitude of the controlled system in bi-directional impulse control is smaller than that of the single direction impulse control system in the steady state, which entails a correct crystal growth. The changes of rotation speed after the different impulse control applied are shown in Figs. 7(c) and 7(d). Figure 7(c) represents the situation where the system rotation speed is controlled by bi-directional impulse, corresponding to Fig. 7(a). The average rotation speed of the system does not change, being kept at $\Omega=1$.1, because the bi-directional impulse control alternatively increases and decreases the speed during the same amount of time. Figure 7(d) depicts the rotation speed after applying the single impulse control, corresponding to Fig. 7(b); in this case, the average rotation speed of the system is reduced, below $\Omega=1$. .

\section{B. The robustness of the method to the small parameter uncertainty}

The proposed method is insensitive to the small model parameter uncertainty in the FSRL system, which is also validated by the corresponding simulations. The reason is as follows. The control parameters are designed according to the bifurcation diagram calculated by the nominal system parameters including $A$ and $c$. These control parameters are robust to small system uncertainties, as made evident by the bifurcation diagram when the parameters are far from the bifurcation points. This fact can be observed from the bifurcation diagrams in Fig. 8. For fixed $\kappa$ and $\Delta$, the period one can be achieved even though there exist small parameters mismatch between the actual system and the controller design. The method is sensitive 


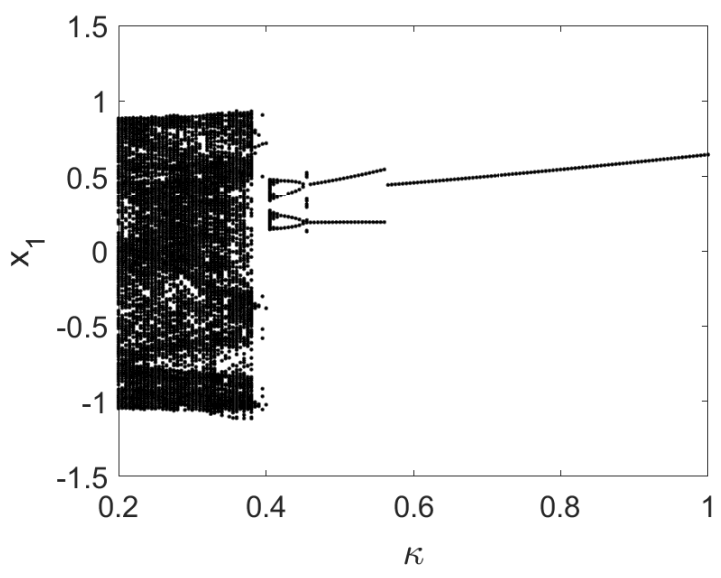

(a)

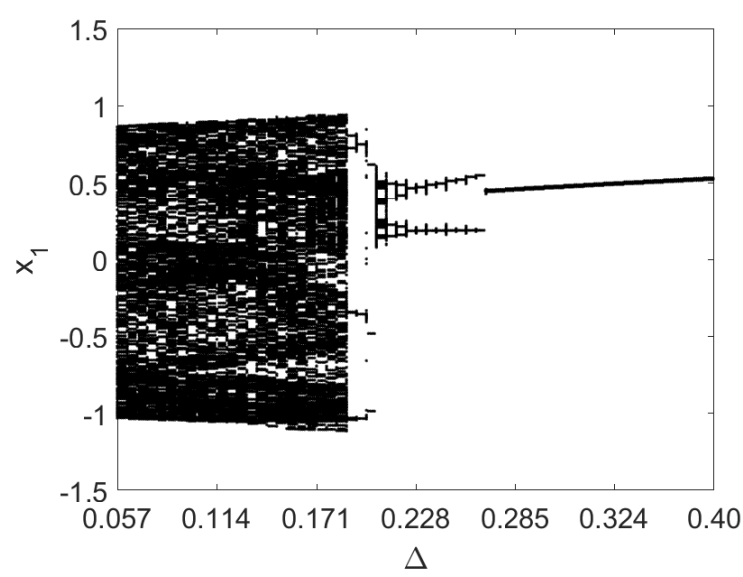

(b)

FIG. 5. (a) The bifurcation diagram of the controlling impulse parameters $\kappa$ and $\Delta$ with $A=0.2, \Omega=1.1, c=0.1$ and $\Delta=0.342$ for varying control parameter $\kappa$. (b) The bifurcation diagram of the system with $A=0.2, \Omega=1.1, c=0.1$ and $\kappa=0.6$ for varying control parameter $\Delta$.

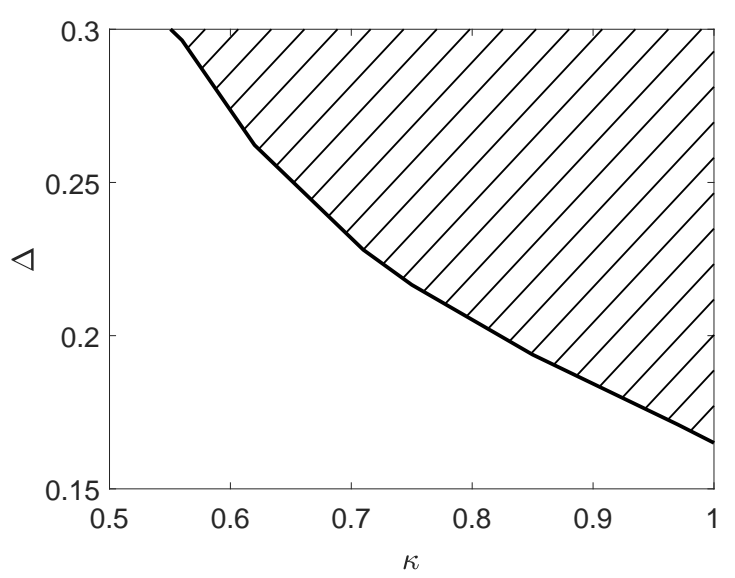

FIG. 6. The pairs of $(\Delta, \kappa)$ in the shade area can be used to suppress chaotic motion.

to the excitation frequency, though, it is fortunate that the excitation frequency is determined by the rotation speed. If the rotation speed is not very accurate, the frequency determined on the same apparatus base is inaccurate accordingly. Therefore, this inaccuracy will not destroy the effectiveness of the proposed method.

Figures 9(a) and 9(b) show the control results with small parameter uncertainty, including $A$ and $c$. We choose the control parameters pair $(\kappa, \Delta)=(0.8,0.257)$ from Fig. 6 and keep the excitation frequency $\Omega=1.1$ unchanged. Figures 9 (a) and 9(b) show that the state variable $x_{1}$ is stabilized in a period one motion after the control is applied, where the model parameters are $(A, c)=(0.19,0.08)$ and $(A, c)=(0.24,0.12)$, respectively. They are all different from the ones used for the controller design in Fig. 6 (i.e., $A=0.2$, c $=0.1$ ). From Figs. 9 , it can be seen that the uncertainty of the system parameter$\mathrm{s}$ does not affect the method for suppressing chaos, once the parameters selected is far from the bifurcation points.

\section{v. CONCLUSIONS}

This work investigates the vibration response of the FSRL system by the perturbation method of multiple scales. A bi-directional impulse control method is proposed to suppress chaos in FSRL system. The advantages of this method is that it ensures the average rotation speed of the system to remain constant, and the amplitude of the resulting period one operation is smaller with respect to the single direction impulse control. Numerical simulations show that the proposed method is an effective control technique for the chaos suppression in the FSRL system, and it is insensitive to the system parameters small uncertainty, including $A$ and $c$. This point is also an important aspect of our proposed bi-directional control for practical application.

\section{DATA AVAILABILITY}

The data that supports the findings of this study are available within the article.

${ }^{1}$ H. P. Ren, Z. X. Zhou, and C. Grebogi, Nonlinear dynamics in the flexible shaft rotating-lifting system of silicon crystal puller using Czochralski method, Nonlinear Dyn. 102, 771-784 (2020).

${ }^{2}$ Z. X. Zhou, C. Grebogi, and H. P. Ren, Parameter impulse control of chaos in crystal growth process, J. Cryst. Growth 563, 126079 (2021).

${ }^{3}$ E. Ott, C. Grebogi, and J. A. Yorke, Controlling Chaos, Phys. Rev. Lett. 64, 1196-1199 (1990).

${ }^{4}$ G. R. Chen, X. N. Dong, On feedback control of chaotic nonlinear dynamic systems, Int. J. Bifurca. Chaos 02, 407-411(1992).

${ }^{5} \mathrm{M}$. T. Yassen, Controlling chaos and synchronization for new chaotic system using linear feedback control, Chaos. Soliton. Fract. 26, 913-920 (2005).

${ }^{6} \mathrm{~K}$. Pyragas, Continuous control of chaos by self-controlling feedback, Phys. Lett. A. 170, 421-428 (1992).

${ }^{7}$ S. Vaidyanathan, C. Volos, Analysis and adaptive control of a novel 3D conservative no-equilibrium chaotic system, Arch. Control Sci. 25, 333353 (2015). 


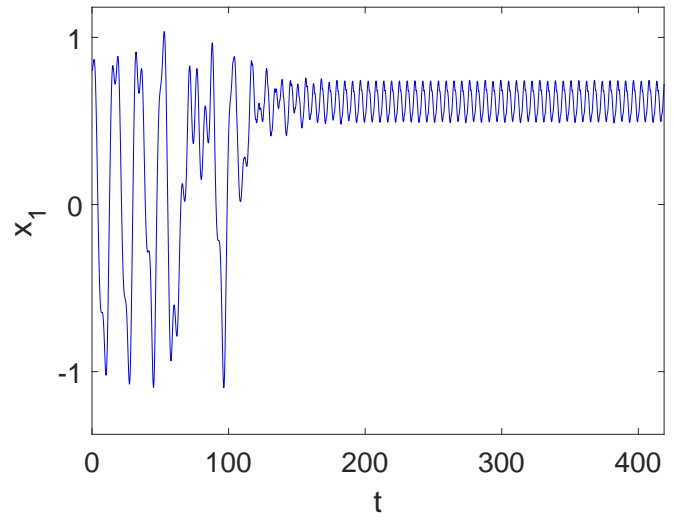

(a)

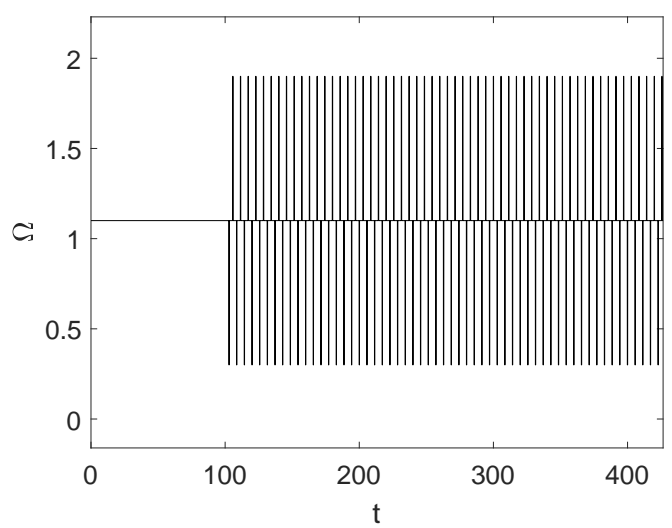

(c)

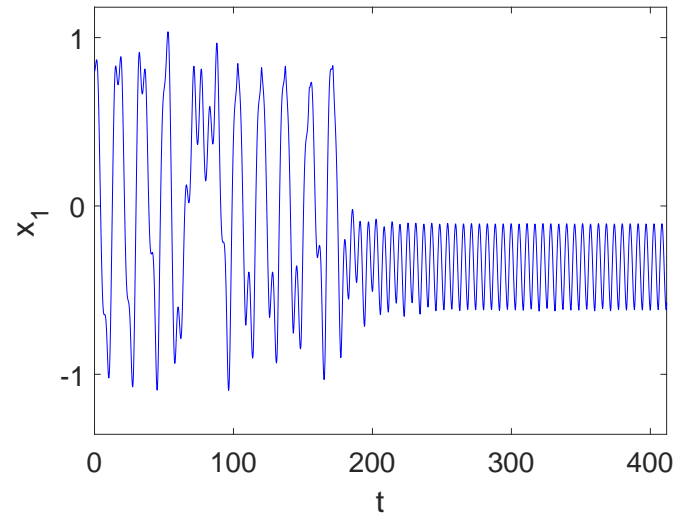

(b)

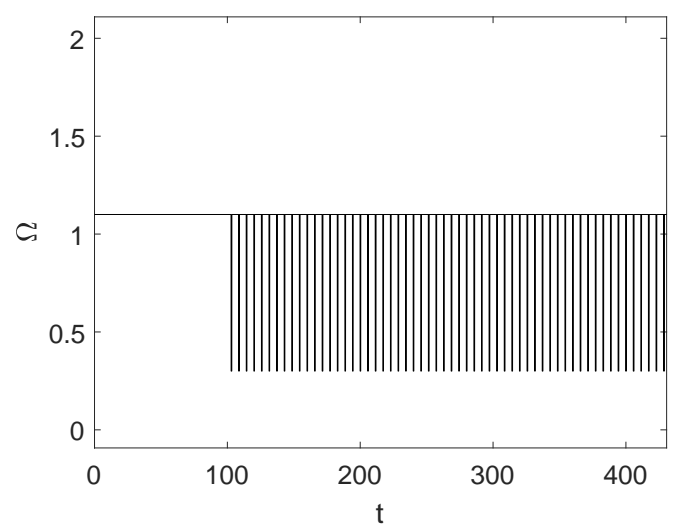

(d)

FIG. 7. (a) The state variable $x_{1}$ as a function of $t$ for $\kappa=0.8$ and $\Delta=0.257$. The bi-directional impulse control is applied at $t=100$; (b) The simulation result of single impulse control by choosing the control parameters pair $(\kappa, \Delta)=(-0.8,0.51)$. The state variable $x_{1}$ of the controlled system in single impulse control is plotted as $t$ varies and the single impulse control is applied at $t=100$; (c) The rotation speed after the bi-directional impulse control is applied; (d) The rotation speed after the single impulse control is applied.

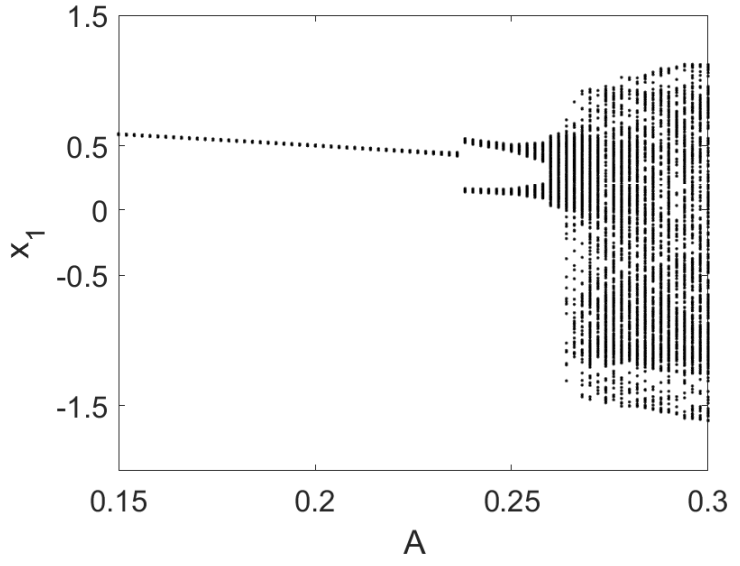

(a)

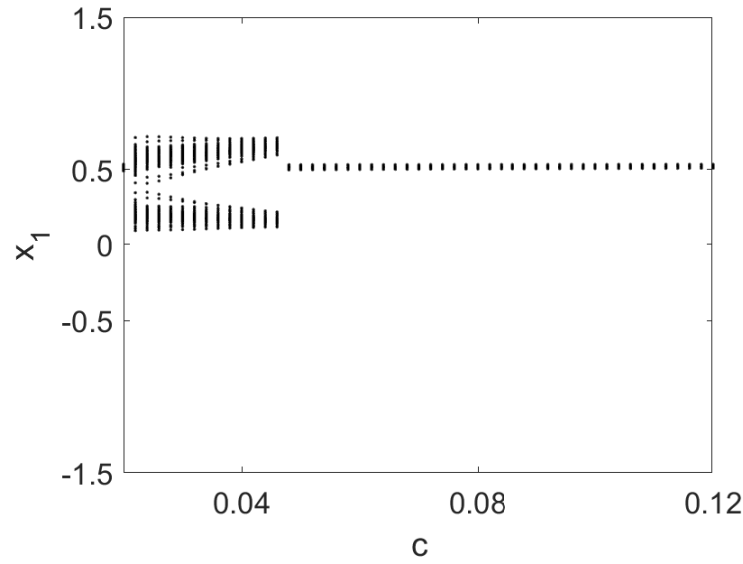

(b)

FIG. 8. (a) The bifurcation diagram of the system with $\Omega=1.1, c=0.1$ and $\kappa=0.8, \Delta=0.257$ by varying $A$, and the period one is achieved at $A \in(0.15,0.24)$. (b) The bifurcation diagram of the system with $\Omega=1.1, A=0.2$ and $\kappa=0.8, \Delta=0.257$ by varying $c$, and the period one is achieved at $c \in(0.05,0.12)$. 


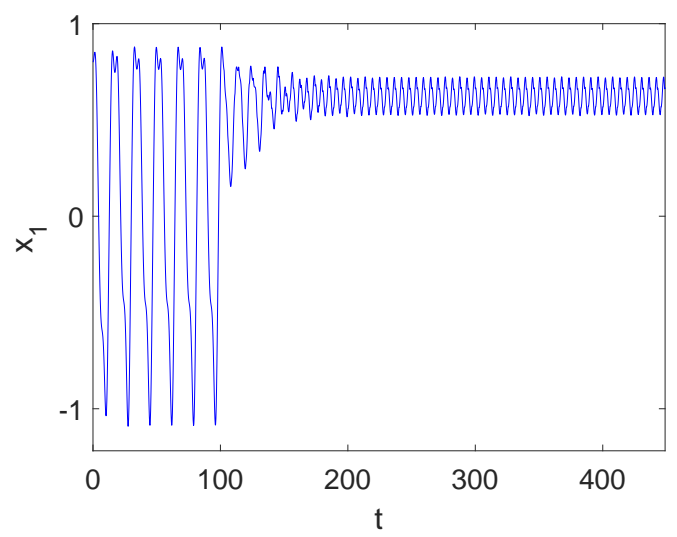

(a)

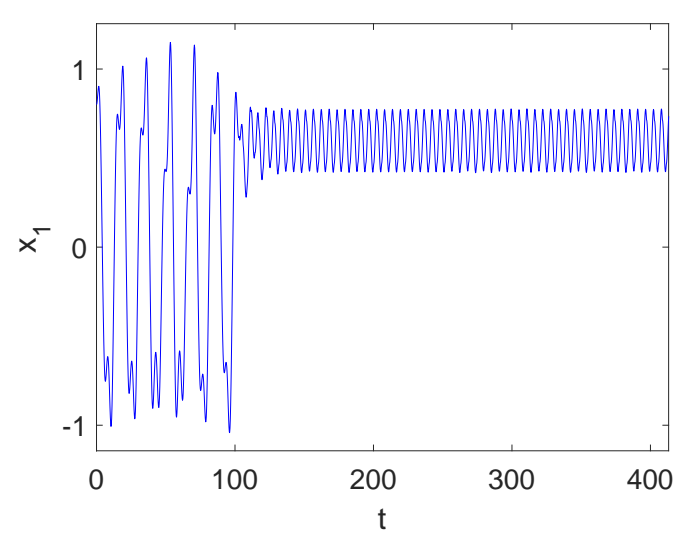

(b)

FIG. 9. (a) The state variable $x_{1}$ is plotted for $\kappa=0.8$ and $\Delta=0.257$, where $\Omega=1.1, A=0.19$ and $c=0.08$. The bi-directional impulse control is applied at $t=100$; (b) The state variable $x_{1}$ is plotted for $\kappa=0.8$ and $\Delta=0.257$, where $\Omega=1.1, A=0.24$ and $c=0.12$. The bi-directional impulse control is applied at $t=100$.

${ }^{8}$ S. H. Luo, S. L. Wu, and R. Z. Gao, Chaos control of the brushless direct current motor using adaptive dynamic surface control based on neural network with the minimum weights, Chaos 25, 073102 (2015).

${ }^{9}$ G. T. He, M. K. Luo, Dynamic behavior of fractional order Duffing chaotic system and its synchronization via singly active control, Appl. Math. Mech. 33, 567-582 (2012).

${ }^{10} \mathrm{~J}$. Hu, Y. Qiu, and H. Lu, Adaptive robust nonlinear feedback control of chaos in PMSM system with modeling uncertainty, Appl. Math. Model. 40, 8265-8275 (2016).

${ }^{11}$ I. Pan, A. Korre, S. Das, and S. Durucan, Chaos suppression in a fractional order financial system using intelligent regrouping PSO based fractional fuzzy control policy in the presence of fractional Gaussian noise, Nonlinear Dyna. 70, 2445-2461 (2012).

${ }^{12}$ V. S. Anishchenko, A. N. Silchenko, and I. A. Khovanov, Synchronization of switching processes in coupled Lorenz systems, Phys. Rev. E, 57, 316322 (1998).

${ }^{13}$ V. S. Anishchenko, Y. I. Boev, The mean Poincaré return time locking: A criterion of chaos induced synchronization, Tech. Phys. Lett. 40, 306-308 (2014).

${ }^{14}$ M. F. Danca, N. Kuznetsov, Parameter Switching Synchronization, Appl. Math. Comput. 313, 94-102 (2017).

${ }^{15} \mathrm{~L}$. Li, Suppressing Chaos of Duffing-Holmes System Using Random Phase, Math. Probl. Eng. 2001, 264-265 (2001).

${ }^{16} \mathrm{Z}$. Luo, J. Shen, Stability of impulsive functional differential equations via the Liapunov functional, Appl. Math. Lett. 22, 163-169 (2009).

${ }^{17} \mathrm{H}$. Li, A. Liu, Asymptotic stability analysis via indefinite Lyapunov functions and design of nonlinear impulsive control systems, Nonlinear AnalHybr. 38, 100936 (2020).

${ }^{18}$ J. L. Mancilla-Aguilar, H. Haimovich, Uniform Input-to-State Stability for Switched and Time-Varying Impulsive Systems, IEEE Trans. Auto. Control 65, 5028-5042 (2020).

${ }^{19}$ T. Yang, L. B. Yang, and C. M. Yang, Impulsive control of Lorenz system, Physica D 110, 18-24 (1997).
${ }^{20}$ L. Zhang, H. Jiang, and Q. Bi, Reliable impulsive lag synchronization foraclass of nonlinear discrete chaotic systems, Nonlinear Dyna. 59, 529-534 (2010).

${ }^{21}$ T. Ma, T. Li, and B. Cui, Coordination of fractional-order nonlinear multiagent systems via distributed impulsive control, Int. J. Sys. Sci. 11, 1-14 (2017).

${ }^{22}$ N. Liu, J. Fang, W. Deng, Z. Wu, and G. Ding, Synchronization for a class of fractional-order linear complex networks via impulsive control, Int. J. Control Autom. 16, 2839-2844 (2018).

${ }^{23}$ K. Tian, C. Bai, H. P. Ren, and C. Grebogi, Hyperchaos synchronization using univariate impulse control. Phys. Rev. E. 100, 052215 (2019).

${ }^{24}$ K. Tian, H. P. Ren, and C. Bai, Synchronization stability and circuit experiment of hyperchaos with time delay using impulse control, IEEE Access 8, 72570-72576 (2020).

${ }^{25}$ A. Jimenez-Triana, K. S. Tang, G. Chen, and A. Gauthier, Chaos control in duffing system using impulsive parametric perturbations, IEEE T. CircuitsII 57, 305-309 (2010).

${ }^{26}$ J. Marão, X. Z. Liu , and A. Figueiredo, Using impulses to control the convergence toward invariant surfaces of continuous dynamical systems. Chaos. Soliton. Fract. 45, 1067-1079 (2012).

${ }^{27}$ H. J. Li, Q. X. Ma, Finite-Time Lyapunov Functions and Impulsive Control Design, Complexity 2020, 1-9 (2020).

${ }^{28} \mathrm{X}$. Li, J. Wu, Stability of nonlinear differential systems with statedependent delayed impulses, Automatica, 64, 63-69 (2016).

${ }^{29} \mathrm{~L}$. Xu, D. Xu, Mean square exponential stability of impulsive control stochastic systems with time-varying delay, Phys. Lett. A 373, 328-333 (2009).

${ }^{30}$ G. Osipov, L. Glatz, H. Troger, Suppressing chaos in the duffing oscillator by impulsive actions, Chaos. Soliton. Fract. 9, 307-321 (1998). 